\title{
命HAD
}

ISSN-L: 2530-5115

DOI: http://doi.org/10.22585/hospdomic.v3i4.84

\section{Redes sociales: Recursos interactivos y la información sobre salud}

\section{Social networks: Interactive resources and health information}

María Sanz-Lorente' ${ }^{1,2}$, Ramón Castejón Bolea'

1. Universidad Miguel Hernández, Departamento de Salud Pública e Historia de la Ciencia, Campus San Joan d'Alacant, Alicante, España.

2. Conselleria de Sanidad Universal y Salud Pública, Centro de Salud Pública de Manises, Manises, España.

Correspondencia/Correspondence

María Sanz-Lorente

msanzlor@gmail.com

Recibido/Received

21.09.2019

Aceptado/Accepted

04.10.2019
Conflicto de Intereses/Competing interest

La autora y el autor del trabajo declaran no tener ningún conflicto de interés

CÓMO CITAR ESTE TRABAJO | HOW TO CITE THIS PAPER

Sanz-Lorente M, Castejón Bolea R. Redes sociales: Recursos interactivos y la información sobre salud. Hosp Domic. 2019;3(4):269-77 


\section{RESUMEN}

Dentro del ámbito sanitario, la evolución de la Web 2.0 también se ha hecho patente, y con el paso de los años se observa un cambio hacia una sociedad cada vez más informada sobre salud en Internet, hasta el punto de adoptar nuevos términos para nuestro vocabulario como el de eSalud. La llegada de las redes sociales (RR. SS.) digitales -consideradas el principal estandarte de este nuevo entorno digital- supuso una nueva vuelta de tuerca en la evolución de la comunicación hipermedia y en los modelos de comunicación tradicionales.

Las RR. SS. y la salud han establecido una fuerte relación. $Y$, es que no hay duda de que los social media están afectando a la forma en la que las personas, de los países con índices de desarrollo humano más altos, gestionan su salud. En el ámbito poblacional, el análisis de patrones y tendencias de las búsquedas en plataformas digitales pueden proporcionar información valiosa sobre el comportamiento relacionado con la salud de las poblaciones.

En la atención domiciliaria paciente y familia pasan de espectadores a protagonistas, asumen una posición más activa tanto en la comunicación como en la toma de decisiones. Se podría pensar que las RR. SS. son dominio exclusivo de la generación más joven. Sin embargo, muchos son los adultos, incluso de edad avanzada, que las han adoptado con entusiasmo para mantenerse al día, así como para relacionarse con sus conocidos y familiares.

Como ya se ha estudiado, las RR. SS. han demostrado su potencial en la provisión remota de supervisión clínica. No obstante, es importante prestar especial atención a tres importantes circunstancias: la proporción del adecuado aprendizaje, el adecuado proceso de uso y su supervisión.

Se puede concluir que en "situaciones ideales" las RR. SS. presentan beneficios para todas las partes implicadas en el cuidado domiciliario de la salud.

Palabras clave: Acceso a la Información; Acceso a Internet; Medios de Comunicación Sociales; Guía de Recursos; Servicios de Atención de Salud a Domicilio.

\section{ABSTRACT}

Within the sanitary scope, the evolution of 2,0 Web also has been made patent, and with the passage of the years a change is observed towards a society more and more informed on health in Internet, until the point to adopt new terms for our vocabulary like the one of eSalud. The arrival of the social digital networks (SSNN) -considered the main standard of this new digital surroundings- supposed a new turn of nut in the evolution of the hypermedia communication and in the traditional models of communication.

The SSNN and health have established a strong relation. In addition, it is that there is no doubt that social media are affecting the form in which the people, of the first world, manage their health. In the population scope, the analysis of patterns and tendencies of the searches in digital platforms can provide valuable information on the behavior related to the health of populations.

In home care patient and family become protagonists instead of spectators, they assume one more a more active position as much in the communication as in the decision-making. It would be possible to think that the SSNN are exclusive dominion of the youngest generation. Nevertheless, many are the adults, even of advanced age, that have adopted them warmly to stay up to the day, as well as to be even related to their well-known and familiar ones.

As it has already been studied, the SSNN have demonstrated their potential in the remote provision of clinical supervision. However, it is important to pay special attention to three important circumstances: the proportion of the suitable learning, the appropriated process of use and its supervision. It can be concluded that in "ideal situations" the SSNN present benefits for all the parts implied in the home care of health.

Keywords: Access to Information; Internet Access; Social Media; Resource Guide; Home Care Services. 


\section{INTRODUCCIÓN}

Internet es sin duda el mayor repositorio de información, al que una población cada vez más familiarizada con las nuevas tecnologías de la comunicación y la información (TIC), puede acceder fácilmente. No es desatinado calificar a la Web 2.0 como el principal medio donde conseguir información sobre salud, permitiendo aproximarse sin restricciones a casi toda la información susceptible de ser transmitida $(1,2)$.

Dentro del ámbito sanitario, la evolución de la Web 2.0 también se ha hecho patente, y con el paso de los años se observa un cambio hacia una sociedad cada vez más informada sobre salud en Internet, hasta el punto de adoptar nuevos términos para nuestro vocabulario como el de eSalud, para aludir a la práctica de cuidados sanitarios apoyada en las TIC (3). Además, la Web 2.0 es a su vez tremendamente influyente en la adopción de estilos de vida saludables o no saludables (4).

La comunicación para la salud es un campo de enorme complejidad que se construye desde una mirada trasdisciplinar. La salud se desarrolla no solo desde el sistema de atención sanitaria, sino también desde la multiplicidad de factores que condicionan la vida cotidiana, por lo que es evidente que la comunicación para la salud es una perspectiva necesaria en muchos ámbitos de la sociedad (5).

La llegada de las redes sociales (RR. SS.) digitales -consideradas el principal estandarte de este nuevo entorno digital, que también aglutina a los blogs, wikis y otros servicios multimedia- supuso una nueva vuelta de tuerca en la evolución de la comunicación hipermedia y en los modelos de comunicación tradicionales. El rápido y exitoso debut de las RR. SS. ha encendido las alarmas en los medios de comunicación tradicionales. El nuevo fenómeno de las RR. SS. suma audiencias millonarias, logra la personalización de los usuarios y rompe con algunas de las barreras de las viejas organizaciones mediáticas. Este nuevo medio de comunicación se cuela ya en las pantallas de millones de internautas como una de las principales fuentes de entretenimiento e información (6).

Como parte de los análisis de este nuevo entorno digital, algunos estudios examinaron su impacto en la comunicación en salud. En tal sentido, se describieron aspectos vinculados al aprovechamiento del potencial de las TIC, señalando la necesidad de adaptación de la comunicación entre profesionales sanitarios y pacientes al nuevo escenario de la denominada eSalud, en un proceso que conduzca a la ampliación de las posibilidades de interacción social y el consiguiente empoderamiento para la salud de los ciudadanos $(7,8)$. Por una parte, se ponen de manifiesto elevados usos y una percepción positiva de los actores sobre estos recursos tecnológicos, por la otra, se identifican desviaciones en los procesos de interacción, flexibilidad y descentralización, como resultado de la calidad de los contenidos (9).

Las redes tienen una amplia y diversa aplicación en el ámbito de la salud. En este sentido, un artículo publicado en el Bulletin of the World Health Organization (10), Ilamaba la atención sobre las potencialidades de estas redes para los trabajadores de la salud y las instituciones. Su autora señalaba que:

...hasta hace poco el modelo de comunicación predominante era de "una autoridad a muchos" (por ejemplo, de una institución de salud al público). Sin embargo, las redes sociales han cambiado el monólogo a un diálogo, donde cualquiera con acceso a las tecnologías de la información e Internet puede ser creador de contenido y comunicador...

Sin embargo, la utilidad de las RR. SS. para la salud no estriba solo en potenciar la comunicación horizontal, algo sumamente atractivo, sino que el análisis automatizado del tráfico de información compartida a través de ellas permite detectar patrones y comportamientos asociados a la búsqueda y suministro de información sobre salud y medicamentos $(11,12)$. Saltan a la vista las potencia- 
lidades de estas aplicaciones para la vigilancia en salud, la planificación de servicios de salud, el desarrollo de programas de intervención, de promoción de salud, entre otros (12).

Tenemos, por tanto, una herramienta que favorece la difusión de la información sobre salud a escala planetaria con características de inmediatez. Podría ser «la piedra filosofal» que muchos profesionales de las ciencias de la salud estaban buscando (1). El conjunto de la Web 2.0 brinda una nueva forma de entender la atención médica, ya que ofrece un medio para ser utilizado por el público, los pacientes y los profesionales de la salud (13).

No obstante, a pesar de la potencialidad que nos ofrece la Web 2.0 para el cuidado de la salud y la ingente información que se ha puesto al alcance de la población, con una oferta y una demanda en continuo aumento, la información sanitaria por sí sola no es suficiente para lograr cambios en los hábitos menos saludables. Para ello es necesario combinar la información con otras ayudas para la toma de decisiones. En todo caso, lo que es seguro es que en el empoderamiento social las personas que poseen las habilidades, capacidades y destrezas específicas, pueden participar en el cambio social (14).

Lo que ya es patente es que Internet se ha convertido en una necesidad para muchos usuarios, a la hora de obtener información sanitaria (15). De hecho, en ciertos sectores de la población, esta forma de informarse se ha convertido en un instrumento fundamental de la transmisión del conocimiento en salud (16).

Hasta hace unos años, los sanitarios eran la fuente más fiable de información sanitaria, pero Internet ha causado una gran revolución por la facilidad y rapidez de acceso a la información sobre salud, y ya no es extraño pensar que un paciente puede estar contrastando la información que le da con la que encuentra en la Red (17).

Las ventajas de la búsqueda de información sobre salud por Internet son $(18,19)$ :

- Ayudar a los pacientes a tomar decisiones de salud más informadas.

- Aumentar la autonomía del paciente.

- Reforzar los mensajes de promoción y prevención así como de adherencia al tratamiento.

Las desventajas serían $(18,19)$ :

- Ser fuente de desinformación debido a la gran variabilidad en la calidad de los contenidos Web.

- Causar ansiedad por las diferencias de consejos, testimonios y opiniones.

- Aumentar las expectativas y demandas de los pacientes respecto a nuevos tratamientos o terapias alternativas.

- Ensanchar las diferencias sociales según el grado de acceso a la Red.

A juicio de Marín-Torres et al. (17), el uso de Internet para buscar información sobre salud presenta más ventajas que inconvenientes en lo que se refiere a la relación médico-paciente, ya que Internet actuaría como un refuerzo de los consejos del médico. Es decir, no reemplaza al médico sino que cambia el tipo de relación, lo cual ya ha sido discutido en otras publicaciones sobre este tema. Hay que tener en cuenta que la incorporación de cualquier tecnología tiene efectos sobre los usuarios que la adoptan, impacta sobre su entorno social y lleva a una adaptación que, para producir el máximo beneficio para todos, debe ser generalizada (20).

Históricamente, la comunicación entre pacientes y médicos se ha basado en encuentros personales (21). El teléfono introdujo un cambio radical en el acceso de los pacientes a sus médicos. A mediados de la década de 1980, se comenzó a utilizar la comunicación a través del correo electrónico (22), principalmente con fines académicos; en la actualidad, constituye una forma de comunicación interpersonal habitual, junto con los sistemas de mensajería instantánea y video- 
conferencias. El mismo fenómeno se verificó con el acceso a Internet, que es sin duda una gran fuente de información sobre salud para médicos y pacientes (23).

Las RR. SS. y la salud han establecido una fuerte relación. Y, es que no hay duda de que los social media están afectando a la forma en la que las personas, principalmente en los países más desarrollados, gestionan su salud.

En el ámbito de la salud, los pacientes encuentran tres principales utilidades en el uso de las RR. SS. (24):

Consulta: Con la llegada de la llamada salud 2.0, cada vez son más los profesionales de la salud que han decidido "abrir consulta" en lugares como Twitter o Facebook. Ambas plataformas (aunque Twitter es algo más indicado para ello) proporcionan la posibilidad de que dichos profesionales pongan su conocimiento -y su tiempo libre- al servicio de sus seguidores, resolviendo las dudas que estos les plantean.

Información: Si ya queda clara la importancia del "Dr. Google", es decir el uso del buscador de Google para resolver dudas sobre salud, mención aparte merece la utilización de las redes sociales con el mismo fin. Aquí más que hacer búsquedas aisladas lo frecuente es hacerse seguidor de perfiles que hablen sobre temas de salud más o menos generales que puedan interesar al usuario. Así, por ejemplo una madre primeriza seguramente se haga seguidora de alguna página de pediatría para recibir consejos sobre cómo cuidar a su hijo; alguien que quiera adelgazar buscará páginas que publiquen con frecuencia consejos para perder peso, etc.

Apoyo: Una de las ventajas de las redes sociales más explotadas por los pacientes es la posibilidad de crear grupos de personas agrupadas por intereses comunes. Esta característica la han aprovechado muchos enfermos para crear grupos de apoyo en redes como Facebook donde poder hablar con otras personas en su misma situación y compartir material de interés sobre su enfermedad.

Ahora bien, si hubiese que destacar dos peligros de las RR. SS. para la salud, estos podrían ser el exceso de ruido y la falta de voces autorizadas.

Nadie controla lo que se dice en las RR. SS., lo que provoca que por ellas se extiendan todo tipo de informaciones inexactas o directamente erróneas; algo que es especialmente grave cuando se trata de información sanitaria como consejos para la salud. Esto unido a la dificultad para diferenciar las fuentes de información fiables hace que el binomio redes sociales y salud sea aun visto con recelos por parte de la comunidad sanitaria; a pesar de que varias experiencias ya han demostrado como el buen uso de estas redes puede mejorar la comunicación médico paciente.

Los profesionales de la salud han sido vistos tradicionalmente como un sector algo reticente al uso de las nuevas tecnologías de la información. Quizás por eso su presencia en las redes sociales sea aun algo deficiente. No obstante, su participación es necesaria si se quiere que realmente internet se convierta en una importante herramienta de salud pública.

En consecuencia, a la hora de diseñar cualquier estrategia de comunicación en salud es necesario pensar en los canales más adecuados para hacer llegar la información a sus destinatarios. Los medios de comunicación de masas han sido tradicionalmente uno de los principales pilares para la emisión de mensajes hacia la población. Actualmente, las redes sociales pueden ser un formato idóneo para las acciones de promoción y protección de la salud (25).

En el ámbito poblacional, el análisis de patrones y tendencias de las búsquedas en plataformas digitales -como Google-, el estudio de como "navega" la gente por Internet para obtener información relacionada con la salud, así como la forma en que se comunican y comparten los contenidos, pueden proporcionar información valiosa sobre el comportamiento relacionado con la salud de las 
poblaciones. Este razonamiento llevó a definir, en el año 2009, el concepto de infodemiología (infodemiology) que incluye aspectos como la infovigilancia (infosurveillance) y, por supuesto, el análisis de las RR. SS. (11). Es un área emergente que complementa las herramientas de salud pública ya existentes y que permiten una vigilancia epidemiológica en tiempo real, detectar y cuantificar disparidades en el acceso a la información sanitaria, analizar la efectividad de las intervenciones educacionales y de campañas de salud o disponer de datos autogenerados por el paciente.

\section{UTILIDAD PARA LA ASISTENCIA DOMICILIARIA}

El cuidado domiciliario constituye hoy una modalidad de atención que permite solventar las dificultades derivadas de la sobreocupación hospitalaria y la cronicidad, los cuales constituyen un problema de interés en los países desarrollados y que pueden ser manejados en el domicilio del paciente como una opción costo-efectiva y segura. Para ello, es necesario buscar estrategias que permitan su desarrollo, gestión de riesgos y modelos de atención, logrando mejorar las condiciones de salud de la población $(26,27)$.

El Hospital a Domicilio ( $\mathrm{HaD}$ ) es una modalidad asistencial consolidada y generalizada, que permite dar una atención al paciente digna, equitativa, próxima, ajustada a sus necesidades, segura y excelente. Contribuye a la sostenibilidad del sistema y acerca la atención especializada al escenario terapéutico natural que es el propio domicilio. La HaD del siglo XXI no se plantea ya exclusivamente como opción asistencial, se posiciona como la mejor alternativa para numerosos procesos médicos y quirúrgicos en un determinado momento evolutivo de los mismos. Y esto es posible porque la $\mathrm{HaD}$ proporciona intensidad de cuidados mediante una atención multidisciplinar centrada en el paciente, humanizando esta atención e integrando al paciente y su familia en los cuidados. Fomenta la continuidad asistencial y es segura, efectiva, eficiente y satisfactoria (28).

En el domicilio, paciente y familia pasan de espectadores a protagonistas, cambia su actitud, asumen una posición más activa tanto en la comunicación como en la toma de decisiones y asumen más responsabilidad y más participación en los cuidados (29). El cuidado tiene entre sus pilares el establecimiento de una buena comunicación con el enfermo, mediada principalmente por el lenguaje verbal. Sin embargo, se sabe que no solo la palabra es mediación en la comunicación. Además, los mensajes pueden ser olvidados, recordados o modificados de acuerdo con el significado que le dan los pacientes según su comprensión o utilidad (30). Por tanto, siempre será aconsejable utilizar herramientas comunicativas que permitan recordar e interpretar las instrucciones de forma correcta.

En el HaD el campo de la telemedicina ha experimentado un importante crecimiento y expansión en el conocimiento y la innovación en los últimos 50 años, siendo las subcategorías más comunes de la telemedicina la consulta remota, la teleradiología y la telepatología. Y, se basaban fundamentalmente en la telefonía móvil (31).

A partir de estas experiencias se ha profundizado en el uso de la tecnología digital y las RR. SS. como componente clave para mejorar la asistencia domiciliaria de la salud, teniendo como finalidad mejorar el soporte al final de la vida, tanto en el paciente mayor como en el paliativo (32).

Se podría pensar que las RR. SS. son dominio exclusivo de la generación más joven. Sin embargo, muchos son los adultos, incluso de edad avanzada, que las han adoptado con entusiasmo para mantenerse al día, así como para relacionarse con sus conocidos y familiares.

El uso de las RR. SS. puede ser muy beneficioso para las personas que saben cómo usarlas. En algunas personas, sobre todo mayores, el aprender algo nuevo a menudo puede ser un desafío. Además, los beneficios del uso de las RR. SS. incluyen la mejora de las capacidades cognitivas, la 
disminución de los sentimientos de soledad y lo obvio, una fuente de entretenimiento. En general, las redes sociales parecen tener un efecto positivo en el bienestar de las personas (8). Se ha demostrado que el uso de internet reduce el riesgo de depresión de las personas mayores hasta en un $33 \%$ (33). Aunque hay que tener presente que el uso de las RR. SS. puede aliviar algunos de estos efectos, pero no es suficiente. Estas personas necesitan pasar tiempo regularmente en la presencia física de otros para evitar los efectos emocionales, mentales y físicos del aislamiento y en el ámbito del cuidado de la salud sentir la proximidad del profesional sanitario. Es decir, las RR. SS. son una herramienta positiva para las personas mayores, los enfermos, las familias y los proveedores de cuidados. Pero es algo que debe abordarse con cierto grado de precaución. Es importante alentar a la interacción con amigos y conocidos de "toda la vida", saber ver más allá de una pantalla y saber aprovechar el tiempo para hacer un poco de todo (33).

Como ya se ha estudiado, las RR. SS. (Skype, Facebook, Twitter, etc.) han demostrado su potencial en la provisión remota de supervisión clínica. No obstante, es importante prestar especial atención a tres importantes circunstancias: la proporción del adecuado aprendizaje, el adecuado proceso de uso y su supervisión (34).

Por todo lo aquí expresado se puede concluir que en "situaciones ideales" las RR. SS. presentan beneficios para todas las partes implicadas en el cuidado domiciliario de la salud (enfermos, familiares, cuidadores, equipo sanitario, etc.), aunque estas RR. SS. como cualquier otra tecnología tienen algunos inconvenientes: la amenaza de la privacidad de los enfermos y su entorno, el respeto a su confidencialidad y la necesidad de una adecuada supervisión. Teniendo estos inconvenientes controlados, las RR. SS. pueden ser un gran apoyo en la hospitalización a domicilio.

Así, de acuerdo a lo expuesto por Barroso y Aguilar (35), la irrupción de la Web Social, dentro de los diferentes ámbitos de nuestra sociedad, resulta un hecho evidente en el siglo XXI. Las herramientas 2.0 se han convertido en el mayor medio de comunicación, colaboración, transmisión y difusión de información, permitiendo interaccionar de forma activa con otros usuarios, transformándose en un espacio en el que prevalece la democratización del conocimiento y aprendizaje común de una forma rápida y cómoda. Todo ello, bajo la "filosofía 2.0", "donde lo esencial no es el volumen de herramientas que se emplea, sino el buen uso que se hace de ellas, pudiendo contribuir, publicar, compartir y colaborar, y evitar ser un simple espectador pasivo». En las redes se puede vivir o sobrevivir, como publicó Fariñas en 2011 (36). Si viven, actúan a conciencia, piensan, generan debates, opinan, disienten, aportan, coinciden, se integran conforme a propósitos deliberados. Si sólo sobreviven, como es el caso de la mayoría de las personas mayores, son actores pasivos, simples caminantes del camino señalado por otros.

\section{BIBLIOGRAFÍA}

1. Sanz-Valero J, Castiel LD, Wanden-Berghe C, Juan Quilis V. Internet y la búsqueda de información en salud pública: desde la relevancia hacia la «revelancia». Gac Sanit. 2006;20(2):159-60. PMID: 16753094

2. Sanz-Lorente M, Guardiola-Wanden-Berghe R. Evaluación de la calidad de las páginas Web sobre el Hospital a Domicilio: el Indicador de Credibilidad como factor pronóstico. Hosp Domic. 2017; 1(2):73-82. DOI: 10.22585/hospdomic.v1i2.11

3. Villaescusa Martínez V, Sáez Villar L. Búsqueda de información sobre salud a través de Internet. eGlobal. 2013;12(3):197-205. 
4. Tonsaker T, Bartlett G, Trpkov C. Health information on the Internet: gold mine or minefield? Can Fam Physician. 2014;60(5):407-8. PMID: 24828994

5. Díaz H. El papel de la comunicación en salud. Viure en Salut. 2014;(100):4-5.

6. Campos Freire F. Las redes sociales trastocan los modelos de los medios de comunicación tradicionales. Rev Lat Comun Soc. 2008;(63):287-93.

7. Bacallao Pino LM. Recursos interactivos y redes sociales en la información sobre salud sexual y reproductiva en la prensa cubana: la sección «sexo sentido» del diario Juventud Rebelde. Rev Cuba Inf Cienc Salud. 2015;26(2):94-106.

8. Torres Valdés RM, Santa Soriano A. Necesidad de adaptar los procesos de comunicación en salud a la Sociedad Red: Nuevas formas relacionales entre profesional sanitario y paciente. Historia y Comunicación Social. 2014;18(Nov):1-17. DOI: 10.5209/rev_HICS.2013.v18.44277

9. Lupiáñez-Villanueva F. Internet y salud: una aproximación empírica a los usos de Internet relacionados con la salud. En: Adicciones y nuevas tecnologías de la información y de la comunicación: Perspectivas de su uso para la prevención y el tratamiento. Logroño, España: Gobierno de La Rioja; 2009. p. 103-18.

10. McNab C. What social media offers to health professionals and citizens. Bull World Health Org. 2009;87(8):566. DOI: 10.2471/blt.09.066712; PMID: 19704998

11. Eysenbach $\mathrm{G}$. Infodemiology and infoveillance: framework for an emerging set of public health informatics methods to analyze search, communication and publication behavior on the Internet. J Med Internet Res. 2009;11(1):e11. DOI: 10.2196/jmir.1157; PMID: 19329408

12. Sánchez Tarragó N. Las redes sociales en Internet y su impacto en la Salud Pública. Rev Cuba Inf Cienc Salud. 2014;25(2):748-58.

13. Moorhead SA, Hazlett DE, Harrison L, Carroll JK, Irwin A, Hoving C. A new dimension of health care: Systematic review of the uses, benefits, and limitations of social media for health communication. J Med Internet Res. 2013;15(4):e85. DOI: 10.2196/jmir.1933; PMID: 23615206

14. Almendro C. Comunidades virtuales de profesionales y pacientes en Mundos Virtuales. En: El ePaciente y las redes sociales [Internet]. Valencia: Instituto Itaca y Fundación Vodafone España; 2011 [citado 20 de mayo de 2019]. p. 133-46. Disponible en: http://bit.ly/2HFsXgu

15. Eysenbach $G$, Köhler $C$. How do consumers search for and appraise health information on the world wide web? Qualitative study using focus groups, usability tests, and in-depth interviews. Br Med J (BMJ). 2002;324(7337):573-7. DOI: 10.1136/bmj.324.7337.573; PMID: 11884321

16. Conesa Fuentes C. Evaluación de la calidad de los sitios Web con información sanitaria en castellano [tesis doctoral]. Murcia, España: Facultad de comunicación y documentación, Universidad de Mucia; 2010.

17. Marin-Torres V, Valverde Aliaga J, Sánchez Miró I, Sáenz del Castillo Vicente MI, PolentinosCastro E, Garrido Barral A. Internet como fuente de información sobre salud en pacientes de atención primaria y su influencia en la relación médico-paciente. Aten Primaria. 2013;45(1):4653. DOI: 10.1016/j.aprim.2012.09.004; PMID: 23140836

18. Wald HS, Dube CE, Anthony DC. Untangling the Web--the impact of Internet use on health care and the physician-patient relationship. Patient Educ Couns. 2007;68(3):218-24. DOI: 10.1016/j. pec.2007.05.016; PMID: 17920226

19. Hartzband P, Groopman J. Untangling the Web--patients, doctors, and the Internet. N Engl J Med. 2010;362(12):1063-6. DOI: 10.1056/NEJMp0911938; PMID: 20335581 
20. Coiera E. Four rules for the reinvention of health care. Br Med J (BMJ). 2004;328(7449):1197-9. DOI: 10.1136/bmj.328.7449.1197; PMID: 15142933

21. Teutsch C. Patient-doctor communication. Med Clin North Am. 2003;87(5):1115-45. PMID: 14621334

22. Mayer Pujadas MA, Leis Machín A. El correo electrónico en la relación médico-paciente: uso y recomendaciones generales. Aten Primaria. 2006;37(7):413-7. DOI: 10.1157/13087384

23. Melamud A, Puiggari J, Goldfarb G, Cairoli H, Otero P. Información sobre salud en Internet: su empleo por pediatras y padres de pacientes. Arch Argent Pediatr. 2007;105(6):513-6.

24. Centro de Empresas de las Tecnologías de la Información y Comunicación (CETIC-UGR). Redes sociales y salud: Para qué sirven y cómo usarlas en tu clínica. Granada, España: CETICUGR; 2019.

25. Revuelta G, Faubel R, Carrasco JM. Comunicación y salud pública. Viure en Salut. 2014;(100):89.

26. Sánchez M, Fuentes G. Gestion clínica de programas de cuidado domiciliario. Rev CES Salud Pública. 2016;7(2):1-11. DOI: 10.21615/cessp.7.2.5

27. Sanz-Lorente M, Castejón-Bolea R. Infografías en las ciencias de la salud: aplicación al cuidado domiciliario. Hosp Domic. 2018;2(2):67-78. DOI: 10.22585/hospdomic.v2i2.44

28. Massa Domínguez B. La hospitalización a domicilio en el siglo XXI. Hosp Domic. 2017;1(1):7-9. DOI: 10.22585/hospdomic.v111.8

29. Gallud Romero J. Por qué sigo creyendo en el hospital a domicilio. Hosp Domic. 2017;1(2):5764. DOI: 10.22585/hospdomic.v1i2.16

30. Madrigal Ramírez MC, Forero Pulido C, Escobar CL. La comunicación, piedra angular en el cuidado de enfermería. Investig Enferm Imagen Desarr. 2013;15(2):49-63.

31. Wang $\mathrm{Y}$, Zhao $\mathrm{Y}$, Zheng J, Zhang A, Dong $\mathrm{H}$. The evolution of publication hotspots in the field of telemedicine from 1962 to 2015 and differences among six countries. J Telemed Telecare. 2018;24(3):238-53. DOI: 10.1177/1357633X17693749; PMID: 28347217

32. Watkins T. Decreasing informal caregiver burden with social media. Home Healthc Nurse. 2014;32(5):304-8. DOI: 10.1097/NHH.0000000000000058; PMID: 24802601

33. Centro Internacional sobre el Envejeciemiento (CENIE). Tercera edad: Las redes sociales también son para ti [Internet]. Salamanca, España: CENIE; 2018 [citado 16 de junio de 2019]. Disponible en: https://cenie.eu/es

34. Bruce T, Byrne F, Kemp L. Using Skype to support remote clinical supervision for health professionals delivering a sustained maternal early childhood programme: a phenomenographical study. Contemp Nurse. 2018;54(1):4-12. DOI: 10.1080/10376178.2018.1441732; PMID: 29455610

35. Barroso Osuna J, Aguilar Gavira S. Las personas mayores y las redes sociales: Un análisis de la situación actual. Aula de Encuentro. 2016;18(1):228-50.

36. Fariñas Díaz JR. El Impacto de las Redes Sociales en la propiedad intelectual. Rev Propiedad Intelectual. 2011;10(14):150-73. 